\title{
COVID-19 Infection in Children with Kidney Transplantation in Iran: A
}

\section{Case Series}

\author{
Shahrbanoo Nakhaei (iD) ${ }^{1}$, Amin Alsadat Sharif (iD ${ }^{2,}{ }^{*}$, Rozita Hoseini Shamsabadi (iD) ${ }^{3}$, Hasan Otukesh (iD) ${ }^{3}$ \\ and Saeide Ghasemi ${ }^{4}$ \\ ${ }^{1}$ Pediatric Gastrointestinal Department, Aliasghar Hospital, Iran University of Medical Sciences, Tehran, Iran \\ ${ }^{2}$ Pediatric Department, School of Medicine, Kashan University of Medical Sciences, Kashan, Iran \\ ${ }^{3}$ Pediatric Nephrology Department, Aliasghar Hospital, Iran University of Medical Sciences, Tehran, Iran \\ ${ }^{4}$ Pediatric Department, Iran University of Medical Sciences, Tehran, Iran \\ "Corresponding author: Pediatric Department, School of Medicine, Kashan University of Medical Sciences, Kashan, Iran. Email: sharifdr4@gmail.com
}

Received 2020 December 06; Revised 2021 July 30; Accepted 2021 August 15.

\begin{abstract}
Introduction: Since mid-April 2020, infection with coronavirus disease of 2019 (COVID-19) has affected more than 2 million people worldwide. Little is known about the effects of COVID-19 infection on kidney transplant patients treated with immunosuppressive (IS) drugs.

Case Presentation: Here, we report three Iranian pediatric kidney transplantation cases who acquired COVID-19 infection and were admitted to Hazrat Ali Asghar Hospital in Tehran, Iran, in March-April 2020 (first wave). They were treated with prednisolone, cyclosporine, and cellcept. All of them had gastrointestinal symptoms, and one patient also had respiratory symptoms. In two patients who had only gastrointestinal symptoms, chest radiographies were considered normal. They did not need the intensive care unit (ICU), ventilator respiratory support, and dialysis due to a temporary increase in serum creatinine and no change in urine volume. Cellcept was stopped and other medications continued. The patient, who had pulmonary and gastrointestinal symptoms, expressed more severe manifestations that led to ICU admission and dialysis. Prednisolone was increased to a stress dose and the other drugs were discontinued.
\end{abstract}

Conclusions: Pulmonary manifestations significantly worsen the prognosis compared to gastrointestinal manifestations in pediatric kidney transplants with COVID-19.

Keywords: COVID-19, Coronavirus, Kidney Transplantation, Immunosuppressive Agents

\section{Introduction}

Coronavirus disease of 2019 (COVID-19) is created by severe acute respiratory syndrome, Coronavirus 2 (SARSCoV2). Children with COVID-19 have a milder disease than adults regarding the severity of symptoms and course of the disease (1-3). However, children undergoing transplantation have a higher morbidity rate if they get infected by COVID-19. This is due to the use of immunosuppressive drugs, which increases the chances of getting various infections $(4,5)$.

According to the Transplant Association, there is little information about transplant patients with COVID-19 (6). Different countries have taken various measures to manage this pandemic, and many centers have stopped all transplant surgeries $(7,8)$. The clinical manifestations vary in children with COVID-19. Fever or chills and cough are the most commonly reported symptoms $(9,10)$. Gastrointestinal symptoms such as nausea, vomiting, and diarrhea $(2,3,11)$ may occur in the absence of respiratory symptoms $(1,10)$, which are less common than pulmonary manifestations (12).

Iran is one of the largest kidney transplant centers in the world (13). In this paper, we report three COVID-19 infected pediatric cases with kidney transplantation. To the best of our knowledge, this report is one of the first case reports of pediatric kidney Transplant recipients with COVID19 in Iran.

\section{Case Presentation}

The clinical manifestation and laboratory data of the cases are shown in Table 1. 


\begin{tabular}{|c|c|c|c|c|c|c|c|c|c|c|c|c|c|c|}
\hline Case & Clinical ManifestAtion & $\begin{array}{l}\text { PCR of EBV, } \\
\text { CMV, BK }\end{array}$ & $\begin{array}{l}\underset{W B C}{\operatorname{Wr}}(\times \\
\left.10^{3} / \mathbf{m m}^{3}\right) \\
\text { Lymph }\end{array}$ & $\begin{array}{c}\mathbf{H B} \\
(\mathrm{gm} / \mathrm{dL})\end{array}$ & $\begin{array}{l}\text { PLT } \\
(\times \\
\left.10^{3} / \mathbf{m}^{3}\right)\end{array}$ & $\begin{array}{l}\text { BUN } \\
(\mathbf{m g} / \mathbf{d L})\end{array}$ & $\begin{array}{c}\text { Cr } \\
\text { (prior-now) } \\
(\mathbf{m g} / \mathbf{d L})\end{array}$ & $\begin{array}{l}\mathrm{HCO} 3 \\
(\mathrm{mEq} / \mathrm{L})\end{array}$ & $\begin{array}{c}\mathrm{Na} \\
(\mathrm{mEq} / \mathbf{L})\end{array}$ & $\underset{(\mathbf{m E q} / \mathbf{L})}{\mathbf{K}}$ & $\underset{(\mathbf{m g} / \mathbf{d L})}{\mathrm{Ca}}$ & $\begin{array}{l}\text { ESR } \\
(\mathrm{mm} / \mathrm{hr})\end{array}$ & $\underset{(\mathrm{mg} / \mathrm{L})}{\mathrm{CRP}}$ & $\begin{array}{l}\text { PCR of } \\
\text { COVID- } \\
19\end{array}$ \\
\hline 1 & $\begin{array}{l}\text { Severe anorexia, nausea, } \\
\text { occasional vomiting, and } \\
\text { malaise }\end{array}$ & Negative & $9.6-9 \%$ & 10 & 277 & 116 & $1.1-2.05$ & 21 & 124 & 2.6 & 9.4 & 63 & 0.8 & Positive \\
\hline 2 & $\begin{array}{l}\text { Fever, malaise, myalgia, and } \\
\text { diarrhea }\end{array}$ & Negative & $3.5-20 \%$ & 10 & 320 & 35 & $0.8-1.5$ & 19 & 136 & 3.9 & 9 & 42 & 10 & Positive \\
\hline 3 & $\begin{array}{l}\text { Fever, cough, mild diarrhea, } \\
\text { severe anorexia, and } \\
\text { respiratory distress }\end{array}$ & Negative & $2.5-30 \%$ & 9 & 140 & 85 & $2.5-5$ & 13 & 130 & 4.9 & 8 & 110 & 23 & Positive \\
\hline
\end{tabular}

\subsection{Case 1}

A 13-year-old boy who had kidney transplantation about two years ago due to focal segmental glomerulosclerosis (FSGS) was admitted to our hospital because of severe anorexia, nausea, occasional vomiting, diarrhea, and malaise. He did not have a cough, rhinorrhea, or respiratory distress. He was on tacrolimus $4 \mathrm{mg}$, mycophenolate mofetil $1000 \mathrm{mg}$ per day, and prednisolone $5 \mathrm{mg}$ every other day. He had no history of recent travel or contact with a known patient with COVID-19. At admission, vital signs were as follows: blood pressure $90 / 50 \mathrm{mmHg}$, pulse rate $120 / \mathrm{min}$, respiratory rate $18 / \mathrm{min}$, and axillary temperature $37.3^{\circ} \mathrm{C}$. Oxygen saturation (SPO2) was $96 \%$ on room air. The patient had moderate dehydration. Chest radiography did not show infiltrations, pulmonary edema, or pleural effusion. Abdominal radiography was normal. Urinalysis did not show proteinuria or hematuria. The patient did not have any change in urine output. Blood, stool, and urine cultures were negative. Electrocardiogram revealed sinus tachycardia. Other physical exams were normal. Lab tests were as follows: (1) creatinine (Cr) $2.05 \mathrm{mg} / \mathrm{dL}$; (2) WBC 9.6 $\times 10^{3} / \mathrm{mm}^{3}$ with $85 \%$ Segment, 3\% Band and 9\% Lymphocyte; (3) hemoglobin $10 \mathrm{gm} / \mathrm{dL}$; (4) platelet $277 \times 10^{3} / \mathrm{mm}^{3}$; (5) erythrocyte sedimentation rate (ESR) $63 \mathrm{~mm} / \mathrm{hr}$; and (6) C-reactive protein $(\mathrm{CRP})<1 \mathrm{mg} / \mathrm{dL}$. Nasopharyngeal PCR was positive for SARS-CoV2.

The patient was hydrated. Hypokalemia and hyponatremia were corrected. Antipyretic was prescribed, and ceftriaxone was given until the cultures were negative. Mycophenolate mofetil (MMF) was stopped, and tacrolimus and prednisolone dosage remained unchanged. Twentyfour hours after admission, the patient felt much well and was discharged after three days. He was asymptomatic at the time of discharge, and serum creatinine returned to pre-infection level $1.1 \mathrm{mg} / \mathrm{dL}$. The patient's diarrhea improved within a week, and MMF was restarted at the previous dose for the patient. The patient was quarantined for two weeks at home. The nasopharyngeal PCR test was repeated after two weeks, and the result was negative.

\subsection{Case 2}

A 16-year-old boy with a history of kidney transplantation from the age of 12 years due to FSGS was admitted to our pediatric nephrology clinic. He complained of fever, malaise, myalgia, and diarrhea for several days. He did not have a cough or respiratory distress. His chest radiography was normal. He had no history of traveling or contacting any patient. At the time of examination, vital signs were as follows: (1) blood pressure 100/70 mmHg; (2) pulse rate $100 / \mathrm{min}$; (3) respiratory rate $19 / \mathrm{min}$; and (4) axillary temperature $38^{\circ} \mathrm{C} . \mathrm{O}_{2}$ saturation was in the normal range, $96 \%$ on room air, and other physical examinations were normal. He was on MMF $1500 \mathrm{mg}$, cyclosporine $150 \mathrm{mg}$ per day, and prednisolone $5 \mathrm{mg}$ every other day. Lab tests were as follows: (1) WBC $3.5 \times 10^{3} / \mathrm{mm}^{3}$ with $20 \%$ lymphocyte; (2) HB 10 gm/dL; (3) Platelets $320 \times 10^{3} / \mathrm{mm}^{3}$; and (4) Cr $1.5 \mathrm{mg} / \mathrm{dL}$. Nasopharyngeal PCR was positive for SARS-CoV2. The treatment was performed as before the case. MMF was stopped until diarrhea recovers and cyclosporine with prednisolone was continued. The patient was discharged on the fourth day with a good general condition and improved diarrhea with a decrease in creatinine to 0.8 . The patient was quarantined at home for two weeks to prevent the spread of the infection. After two weeks, Nasopharyngeal PCR for COVID-19 was negative.

\subsection{Case 3}

An 18-year-old boy with a history of kidney transplantation for five years due to a neurogenic bladder was admitted to our pediatric nephrology clinic. He had a fever, cough, mild diarrhea, and severe anorexia for two weeks. At the time of admission, he had respiratory distress. SPO2 was $82 \%$ on room air, which increased to $95 \%$ with supplemental oxygen using a face mask. Physical examination showed tenderness at the transplanted kidney site, conjunctivitis in both eyes, mild dehydration, and crackles in the right lower lung. Urine output decreased to $0.4 \mathrm{cc} / \mathrm{kg} /$ hour. In primary lab tests, WBC was $2.5 \times$ $10^{3} / \mathrm{mm}^{3}$, with $30 \%$ lymphocyte, HB $9 \mathrm{mg} / \mathrm{dL}$, platelets 140 
$\times 10^{3} / \mathrm{mm}^{3}$, Cr $5 \mathrm{mg} / \mathrm{dL}$, and (pre-infection serum creatinine was $2.5 \mathrm{mg} / \mathrm{dL}$ ). Nasopharyngeal PCR was positive for SARS-CoV2. At the time of admission, while Chest X-Ray (CXR) had no specific findings in the patient, the chest computed tomography (CT) scan (Figure 1) showed the pattern of COVID-19 pneumonia in the form of groundglass opacities in both lungs. He was admitted to our nephrology ward. Initially, ceftriaxone was initiated for the patient. However, on the third day, due to deterioration of the patient's respiratory status and general condition, he was transferred to the intensive care unit (ICU) for cardiopulmonary monitoring and hemodialysis, and broad-spectrum antibiotics were prescribed and continued throughout the patient's hospitalization despite all the negative cultures in terms of the virus, bacteria, and fungi. Lopinavir-ritonavir was then initiated. MMF and cyclosporine were stopped, and corticosteroid increased and changed to a stress dosage. Since acute febrile illness causes minor stress, $30-50 \mathrm{mg} / \mathrm{m}^{2} /$ day of hydrocortisone (this amount increased $3-5$ times in case prednisolone was used) was given to the patient until the fever stopped, and then, the drug was tapered and returned to the patient's previous consumption (14). The respiratory condition was improved after one week, and the patient was transferred to the ward. However, the graft was lost and he underwent dialysis.

\section{Discussion}

Here, we reported three 13 - 18-year-old Iranian pediatric kidney transplantation cases who acquired COVID19. Based on our study, pulmonary manifestations significantly worsen the prognosis compared to gastrointestinal manifestations in pediatric kidney transplants with COVID-19. COVID-19 can affect children and adults and is more severe in people with underlying diseases. The most important action in children with COVID-19 is supportive care. Among these, the respiratory system is a priority (15). No antiviral drug was specifically designed for the treatment of diarrhea (16).

Some studies discussed the outcome of COVID-19 in kidney transplant recipients. One short series (17) reported eight cases of kidney transplant recipients infected with COVID-19 (median age $=48.5$ years; range $=21-71$ years), including four males and four females. The most common presenting features were fever and cough. One patient was managed on an outpatient and the remaining seven patients were hospitalized. One of the hospitalized patients was admitted to intensive therapy and underwent mechanical ventilation and continuous Veno-Venous Haemodiafiltration (CVVH).
Kidney transplant recipients take immunosuppressive drugs throughout their lives. Dose monitoring of immunosuppression (IS) is essential to balance the risk of transplant rejection and infection. Immunosuppressive therapy management is still unknown in pediatric kidney transplant patients with COVID-19. After transplantation, immune system suppression, especially calcineurin inhibitors (CNIs), reduces the adaptive $T$ cell response and thus increases the spread of the virus. On the other hand, coronavirus $\mathrm{N}$ protein is a multifunctional protein required for virus replication, and non-suppressive derivatives of the cyclosporine reduce the expression of this protein (18).

Children with COVID-19 have milder respiratory symptoms than adults, or they may even be asymptomatic (13). Although pulmonary manifestation is the most common presentation in children with SARS-CoV2 infection, gastrointestinal manifestations such as diarrhea may also occur in them (19). In a study conducted by Rahimzadeh (20) on nine children with COVID-19, all children had at least one infected family member. Fever, chills, myalgia, cough, tachypnea, retraction, and crackle were common in all cases. None of the patients presented with diarrhea and vomiting. At least this study showed COVID-19 pneumonia prognosis was good in children with no underlying diseases and the patients recovered without the need for $\mathrm{LPN} / \mathrm{r}$, ribavirin, or mechanical ventilation. Kidney is another organ involved in the Covid-19 pandemic. COVID-19 can cause acute kidney injury (AKI) in kidney transplant recipients (21). In a study conducted in the same field on seven adults, four of them had AKI (57\%); three people needed dialysis and one died (22). A study by Amina showed that COVID-19 can cause kidney damage and proteinuria was associated with an increased risk of AKI and hematuria was associated with ICU admission, intramechanical ventilation, and death (23).

A cross-sectional study was conducted on 71 pediatric patients with COVID-19 infection. On admission, $10 \%$ of patients had oliguria, 7.7\% had edema, and 3\% had hypertension. The first urinalysis indicated proteinuria, leukocyturia, and hematuria in 46,24 , and $23 \%$ of the patients, respectively. Overall, $40.7 \%$ of the patients showed some degree of kidney involvement during hospitalization, and AKI occurred in $34.5 \%$ of the patients (24). The mechanism of this damage can be virus interaction with the Angiotensin Converting Enzyme-2 (ACE2) receptor in proximal renal tubules and glomeruli (25). However, the most common cause of diarrhea after transplant is infections, but it can also be due to medications. Since MMF can cause ulcerative colitis, it accounts for approximately 50\% of drug-induced diarrhea (10-12). 


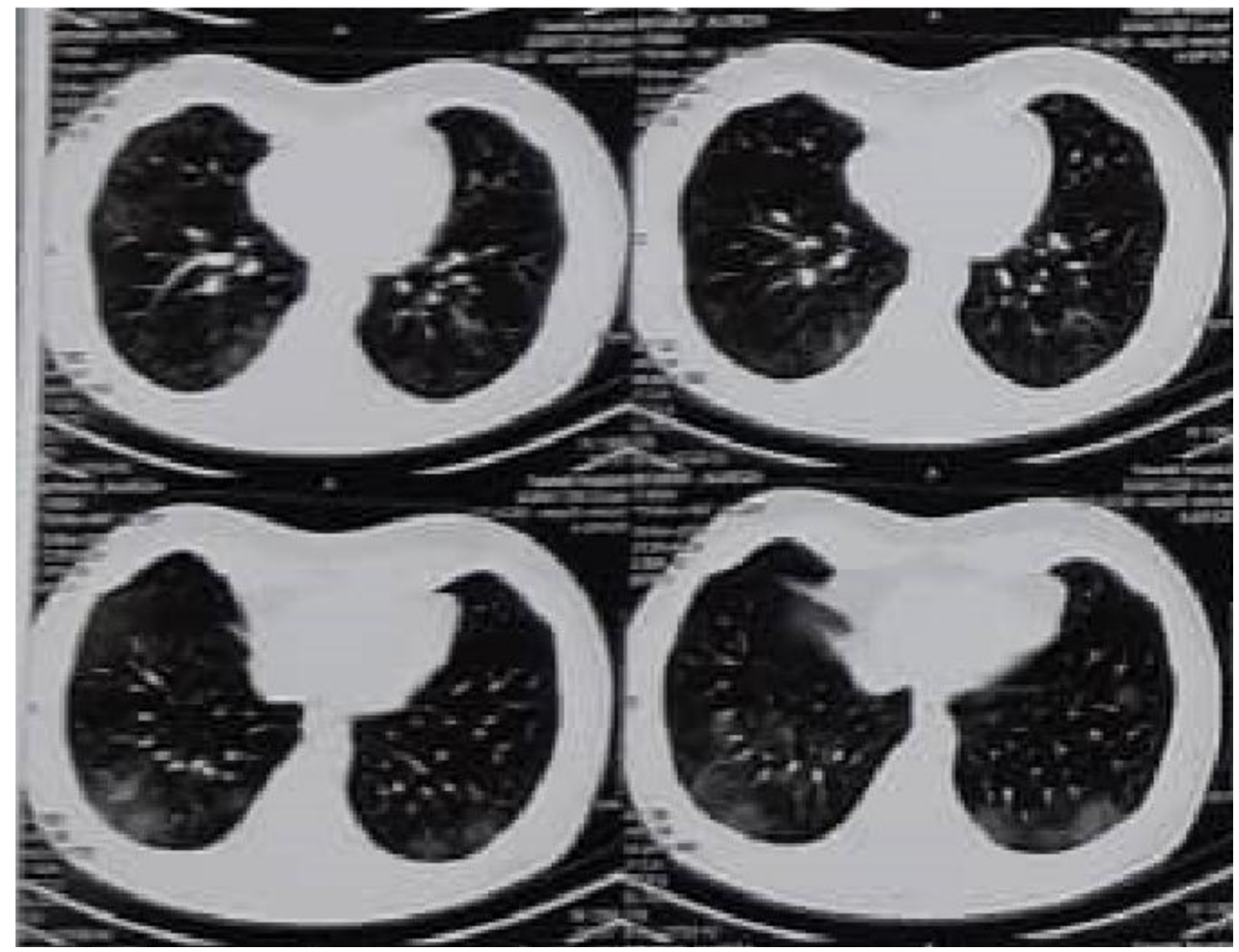

Figure 1. Chest CT of case 3

Management of immunosuppressive drugs in transplant recipients infected with COVID-19 is challenging. Although it may appear reasonable to reduce or discontinue immunosuppressive drugs to fight infections, due to the activation of the inflammatory cascade caused by COVID19 (26), the administration of immunosuppressive drugs can be useful (13). However, this must be proven (27). According to studies reported so far, kidney transplant recipients with mild COVID-19 should receive their CNI and glucocorticoids as before, but their anti-proliferative drugs such as MMF should be discontinued. However, in patients who have severe symptoms that require ICU and ventilator respiratory support, CNIs and anti-proliferative drugs should be discontinued immediately, and glucocorticoid doses may be increased (28). Common bacterial infections were reported at admission in 3.5\% of COVID-19 patients, while up to $15 \%$ of secondary bacterial infections occurred during hospitalization. To support the limited use of antibiotics, cultures should be sent immediately, and antibiotics should be discontinued if cultures are negative and there are no signs of bacterial pathogens. Antibacterial therapy should be continued for patients with nosocomial pneumonia, secondary bacterial respiratory infection and under ventilators (29).

A recent study (30) showed that lopinavir (LPV) inhibited SARS-CoV2 while the other antiviral drugs (such as ribavirin, favipiravir, oseltamivir, or baloxavir) showed no laboratory effect at concentrations below $100 \mu$ mol. Public health England's recommendation is to use LPV to treat SARS and the Middle East respiratory syndrome (MERS) (31, 32) because it is available and has a favorable toxicity profile. Therefore, it is used in the treatment of patients with COVID-19. LPV is an antiretroviral protease inhibitor and is usually given in combination with low booster doses of ritonavir because it reduces hepatic metabolism by inhibiting cytochrome P450 3A4 enzyme, thereby increasing the effectiveness of LPV(33).

In patients with reduced immunosuppressive drugs, the time to return to the previous dose is also challenging and needs further studies. This report provided a brief overview of the clinical course of COVID-19 in a patient receiving an immunosuppressive drug. A retrospective cohort study was done on 22 pediatric kidney transplant populations at a New York transplant center. Patients had a positive test (Ab or PCR) for SARS-CoV-2 and a comparison between COVID-positive and COVID-negative trans- 
plant patients was performed. Results showed that pediatric kidney transplant recipients are at risk for the development of COVID-19 infection. While this population may be more at risk for SARS-CoV-2 infection due to their immunosuppressed status, their clinical course appears mild and similar to a healthy pediatric population (34). Fiftyone patients underwent transplantation including 11 kidney and 40 liver recipients. In the three months of followup, two patients presented with symptoms including highgrade fever, malaise, rhinorrhea, and GI symptoms. Both patients had two negative PCR for COVID-19, and no radiologic or laboratory results regarding COVID-19 were also detected. Therefore, transplant programs can continue their activities during the COVID-19 epidemic disease by selecting a specific case and following protective protocols (35).

Therefore, it is thought that just as the incidence of COVID-19 is lower and milder in healthy children than in adults, it is also lower in children with immunodeficiency and transplant recipients than in the same adult population. Since our hospital is a referral center and the first center to provide information about COVID-19 infection in children with kidney transplantation in Iran, the small number of patients shows that children are less involved in the disease than adults. Therefore, to improve the treatment cases, the experiences of other nephrologists and infectious disease specialists should be used, both inside and outside the country.

\subsection{Conclusion}

Gastrointestinal symptoms are an uncommon manifestation of COVID-19 in children with a good prognosis, even in patients with underlying diseases. Pulmonary involvement caused by COVID-19 also has a good prognosis in children. However, the prognosis is inferior in patients with underlying diseases requiring ventilator respiratory support. Therefore, studies show that COVID-19 can affect children, like adults. The question that arises is why this disease affects children less severely. By answering this question, a solution can be found to treat this disease. Another question is that why this disease is more prevalent in males, according to various studies, including ours.

\section{Acknowledgments}

The authors would like to express their gratitude to Ali Asghar Clinical Research Development Center (AACRDC).

\section{Footnotes}

Authors' Contribution: Study concept and design, SH. N., and A. SH.; Analysis and interpretation of data, A. SH.;
Drafting of the manuscript, A. SH.; Critical revision of the manuscript for important intellectual content, R. H., H. O., SH. N, and S. GH.

Conflict of Interests: The authors declare that there is no conflict of interest.

Ethical Approval: The study was performed in compliance with the World Medical Association Declaration of Helsinki on Ethical Principles for Medical Research Involving Human Subjects, and Ethical approval was elicited from the Ethical Committee at the Iran University of Medical Sciences in Iran.

Funding/Support: None to declare.

Informed Consent: All patients signed written informed consent for publishing the clinical manifestations and lab data and this study is in accordance with the Helsinki Declaration.

\section{References}

1. Dong Y, Mo X, Hu Y, Qi X, Jiang F, Jiang Z, et al. Epidemiology of COVID19 among children in China. Pediatrics. 2020;145(6). e20200702. doi: 10.1542/peds.2020-0702.

2. Lu X, Zhang L, Du H, Zhang J, Li YY, Qu J, et al. SARS-CoV-2 Infection in Children. N Engl J Med. 2020;382(17):1663-5. doi: 10.1056/NEJMc2005073. [PubMed: 32187458]. [PubMed Central: PMC7121177].

3. Wei M, Yuan J, Liu Y, Fu T, Yu X, Zhang ZJ. Novel Coronavirus infection in hospitalized infants under 1 year of age in China. JAMA. 2020;323(13):1313-4. doi: 10.1001/jama.2020.2131. [PubMed: 32058570]. [PubMed Central: PMC7042807].

4. Avery RK, Fishman JA. Infectious diseases in transplantation. In: Hricik D, editor. Primer on transplantation. 3rd ed. New Jersey, USA: American Society of Transplantation; 2011. p. 51-72. doi: 10.1002/9781444391770.ch4.

5. Fishman JA. Infection in solid-organ transplant recipients. New Eng J Med.2007;357(25):2601-14. doi:10.1056/NEJMra064928.

6. Fishman JA. Guidance on Coronavirus disease 2019 (COVID-19) for transplant clinicians. Montréal, Canada: Transplant Infectious Disease; 2020. Available from: https://tts.org/tid-about/tid-presidentsmessage/23-tid/tid-news/657-tid-update-and-guidance-on-2019novel-coronavirus-2019-ncov-for-transplant-id-clinicians.

7. Syed SM, Gardner J, Roll G, Webber A, Mehta N, Shoji J, et al. COVID19 and abdominal transplant: A stepwise approach to practice during pandemic conditions. Transplantation. 2020;104(11):2215-20. doi: 10.1097/TP.0000000000003387. [PubMed: 32639408]. [PubMed Central: PMC7363383].

8. Ahn C, Amer H, Anglicheau D, Ascher NL, Baan CC, Battsetset G, et al. Global transplantation COVID report March 2020. Transplantation. 2020;104(10):1974-83. doi: 10.1097/TP.0000000000003258. [PubMed: 32243281]. [PubMed Central: PMC7188045].

9. Liguoro I, Pilotto C, Bonanni M, Ferrari ME, Pusiol A, Nocerino A, et al. SARS-COV-2 infection in children and newborns: A systematic review. Eur J Pediatr. 2020;179(7):1029-46. doi: 10.1007/s00431-020-03684-7. [PubMed: 32424745]. [PubMed Central: PMC7234446].

10. Hoang A, Chorath K, Moreira A, Evans M, Burmeister-Morton F, Burmeister F, et al. COVID-19 in 7780 pediatric patients: A systematic review. EClinicalMedicine. 2020;24:100433. doi: 10.1016/j.eclinm.2020.100433. [PubMed: 32766542]. [PubMed Central: PMC7318942]. 
11. CDC Covid- Response Team. Coronavirus disease 2019 in children United States, February 12-April 2, 2020. MMWR Morb Mortal Wkly Rep. 2020;69(14):422-6. doi: 10.15585/mmwr.mm6914e4. [PubMed: 32271728]. [PubMed Central: PMC7147903].

12. Zhang W, Du RH, Li B, Zheng XS, Yang XL, Hu B, et al. Molecular and serological investigation of 2019-nCoV infected patients: Implication of multiple shedding routes. Emerg Microbes Infect. 2020;9(1):3869. doi: 10.1080/22221751.2020.1729071. [PubMed: 32065057]. [PubMed Central: PMC7048229].

13. Malek-Hosseini SA, Habibzadeh F, Nikeghbalian S. Shiraz organ transplant center: The largest liver transplant center in the world. Transplantation. 2019;103(8):1523-5. doi: 10.1097/TP.0000000000002581. [PubMed: 31348432].

14. Kinnett K, Noritz G. The PJ Nicholoff steroid protocol for duchenne and becker muscular dystrophy and adrenal suppression. PLoS Curr. 2017;9. doi: 10.1371/currents.md.d18deef7dac96ed135e0dc8739917b6e. [PubMed: 28744411]. [PubMed Central: PMC5505768].

15. Xia W, Shao J, Guo Y, Peng X, Li Z, Hu D. Clinical and CT features in pediatric patients with COVID-19 infection: Different points from adults. Pediatr Pulmonol. 2020;55(5):1169-74. doi: 10.1002/ppul.24718. [PubMed: 32134205]. [PubMed Central: PMC7168071].

16. D’Amico F, Baumgart DC, Danese S, Peyrin-Biroulet L. Diarrhea during COVID-19 infection: Pathogenesis, epidemiology, prevention, and management. Clin Gastroenterol Hepatol. 2020;18(8):1663-72. doi: 10.1016/j.cgh.2020.04.001. [PubMed: 32278065]. [PubMed Central: PMC7141637].

17. Elhadedy MA, Marie Y, Halawa A. COVID-19 in renal transplant recipients: Case series and a brief review of current evidence. Nephron. 2021;145(2):192-8. doi: 10.1159/000512329. [PubMed: 33291120]. [PubMed Central: PMC7801980].

18. Ma-Lauer Y, Zheng Y, Malesevic M, von Brunn B, Fischer G, von Brunn A. Influences of cyclosporin A and non-immunosuppressive derivatives on cellular cyclophilins and viral nucleocapsid protein during human coronavirus 229E replication. Antiviral Res. 2020;173:104620. doi: 10.1016/j.antiviral.2019.104620. [PubMed: 31634494]. [PubMed Central: PMC7114175].

19. Pan L, Mu M, Yang P, Sun Y, Wang R, Yan J, et al. Clinical characteristics of COVID-19 patients with digestive symptoms in Hubei, China: A descriptive, cross-sectional, multicenter study. Am J Gastroenterol. 2020;115(5):766-73. doi:10.14309/ajg.0000000000000620.[PubMed: 32287140]. [PubMed Central: PMC7172492].

20. Rahimzadeh G, Ekrami Noghabi M, Kadkhodaei Elyaderani F, Navaeifar MR, Enayati AA, Manafi Anari A, et al. COVID-19 infection in Iranian children: A case series of 9 patients. J Pediatr Rev. 2020:139-44. doi: 10.32598/jpr.8.2.139.

21. Hooman N, Sadeghian M, Jahangiri F, Hosseini S. The prevalence and incidence of atypical hemolytic uremic syndrome in Iran: A systematic review and meta-analysis protocol study. J Compr Ped. 2017;9(1). doi: $10.5812 /$ compreped.56080.

22. Banerjee D, Popoola J, Shah S, Ster IC, Quan V, Phanish M. COVID-19 infection in kidney transplant recipients. Kidney Int.2020;97(6):1076-82. doi:10.1016/j.kint.2020.03.018. [PubMed: 32354637]. [PubMed Central: PMC7142878].

23. Chaudhri I, Moffitt R, Taub E, Annadi RR, Hoai M, Bolotova O, et al. Association of proteinuria and hematuria with acute kidney injury and mortality in hospitalized patients with COVID-19. Kidney Blood Press Res. 2020;45(6):1018-32. doi: 10.1159/000511946. [PubMed: 33171466].

24. Mohkam M, Mirzaee M, Abdollah Gorgi F, Rafiei Tabatabaei S, Karimi A, Armin S, et al. Renal involvement in COVID-19 among Iranian children. Arch Pediatr Infect Dis. 2021;In Press(In Press). doi:10.5812/pedinfect.106597.

25. Li W, Moore MJ, Vasilieva N, Sui J, Wong SK, Berne MA, et al Angiotensin-converting enzyme 2 is a functional receptor for the SARS coronavirus. Nature. 2003;426(6965):450-4. doi: 10.1038/nature02145. [PubMed: 14647384]. [PubMed Central: PMC7095016].

26. Nakhaei S, Talachian E, Bidari A. Familial Mediterranean fever: Unusual age of presentation and the role of genetic diagnosis. Arch Iran Med. 2005;8(1):56-9.

27. D'Antiga L. Coronaviruses and immunosuppressed patients: The facts during the third epidemic. Liver Transpl. 2020;26(6):832-4. doi: 10.1002/lt.25756. [PubMed: 32196933].

28. Kronbichler A, Gauckler P, Windpessl M, Il Shin J, Jha V, Rovin $\mathrm{BH}$, et al. COVID-19: Implications for immunosuppression in kidney disease and transplantation. Nat Rev Nephrol. 2020;16(7):365-7. doi: 10.1038/s41581-020-0305-6. [PubMed: 32409782]. [PubMed Central: PMC7224161].

29. Sieswerda E, de Boer MGJ, Bonten MMJ, Boersma WG, Jonkers RE, Aleva RM, et al. Recommendations for antibacterial therapy in adults with COVID-19 - an evidence based guideline. Clin Microbiol Infect. 2021;27(1):61-6. doi: 10.1016/j.cmi.2020.09.041. [PubMed: 33010444]. [PubMed Central: PMC7527308].

30. Choy KT, Wong AY, Kaewpreedee P, Sia SF, Chen D, Hui KPY, et al. Remdesivir, lopinavir, emetine, and homoharringtonine inhibit SARS-CoV-2 replication in vitro. Antiviral Res. 2020;178:104786. doi: 10.1016/j.antiviral.2020.104786. [PubMed:32251767]. [PubMed Central: PMC7127386].

31. Yao TT, Qian JD, Zhu WY, Wang Y, Wang GQ. A systematic review of lopinavir therapy for SARS coronavirus and MERS coronavirus-A possible reference for coronavirus disease-19 treatment option. J Med Virol. 2020;92(6):556-63. doi: 10.1002/jmv.25729. [PubMed: 32104907]. [PubMed Central: PMC7217143].

32. Ford N, Vitoria M, Rangaraj A, Norris SL, Calmy A, Doherty M. Systematic review of the efficacy and safety of antiretroviral drugs against SARS, MERS or COVID-19: Initial assessment. J Int AIDS Soc. 2020;23(4). e25489. doi: 10.1002/jia2.25489. [PubMed: 32293807]. [PubMed Central: PMC7158851].

33. Meini S, Pagotto A, Longo B, Vendramin I, Pecori D, Tascini C. Role of lopinavir/ritonavir in the treatment of Covid-19: A review of current evidence, guideline recommendations, and perspectives. J Clin Med. 2020;9(7). doi: 10.3390/jcm9072050. [PubMed: 32629768]. [PubMed Central: PMC7408758].

34. Singer PS, Sethna C, Molmenti E, Fahmy A, Grodstein E, CastellanosReyes L, et al. COVID-19 infection in a pediatric kidney transplant population: A single-center experience. Pediatr Transplant. 2021;25(4). e14018. doi: 10.1111/petr.14018. [PubMed: 33813782]. [PubMed Central: PMC8250351].

35. Shafiekhani M, Kazemi K, Bahador A, Imanieh MH, Karimzadeh P. Pediatric solid organ transplantation in the era of COVID-19: A follow-up study. Research Square. 2021;Preprint. doi: 10.21203/rs.3.rs-236136/v1. 